Pacific Journal of Mathematics

AUTOMORPHISMS OF THE SEMIGROUP OF FINIS
COMPLEXES OF A PERIODIC LOCALLY CYCLIC GRO

RICHARD DOWEL BYRD, JUSTIN THOMAS LLOYD
FrankLIN D. PEDERSEN AND JAMES WILSON STEP 


\title{
AUTOMORPHISMS OF THE SEMIGROUP OF FINITE COMPLEXES OF A PERIODIC LOCALLY CYCLIC GROUP
}

\author{
RICHARD D. BYRD, JUSTIN T. LLOYd, \\ Franklin D. Pedersen \\ AND JAMES W. STEPP
}

In this paper the automorphism group of the semigroup of finite complexes of a periodic locally cyclic group is determined.

1. Introduction. Let $G$ be a group, written additively but not assumed to be abelian, and let $F(G)$ denote the collection of finite nonempty subsets of $G$. Then $F(G)$ is a semigroup with respect to the operation $A+B=\{a+b \mid a \in A$ and $b \in B\}$. The collection of automorphisms of $F(G)$ is a group under the operation of composition of functions and we shall denote this group by Aut $F(G)$. The automorphism group of $G$ will be denoted by Aut $G$. Since the collection of singleton subsets of $G$ is the group of units of $F(G)$, we frequently identify $G$ with $\{\{g\} \mid g \in G\}$. Each automorphism of $G$ induces an automorphism of $F(G)$ in the natural way. These elements of Aut $F(G)$ will be called standard automorphisms. If $\alpha \in$ Aut $G$ and $\alpha^{*}$ is the standard automorphism of $F(G)$ induced by $\alpha$, then the mapping which sends $\alpha$ to $\alpha^{*}$ is an isomorphism of Aut $G$ onto the collection of standard automorphisms of $F(G)$.

Our interest in Aut $F(G)$ comes from our study of retractable groups. In [1] it was shown that the retractions of a torsion-free abelian group $G$ generate a large class of nonstandard automorphisms of $F(G)$. In particular, it was shown that Aut $F(Z)$ is countably infinite, where $Z$ denotes the additive group of integers. Since Aut $Z$ has only two elements, it was natural to inquire if the semigroup of complexes of a finite cyclic group admits nonstandard automorphisms. For a natural number $n$, let $Z_{n}$ denote the group of integers modulo $n$. Clearly Aut $F\left(Z_{1}\right)$ and Aut $F\left(Z_{2}\right)$ have only standard automorphisms. In $\S 3$ we exhibit nonstandard automorphisms for $F\left(Z_{3}\right), F\left(Z_{4}\right)$, and $F\left(Z_{5}\right)$ and classify their corresponding automorphism groups. The only automorphisms of $F\left(Z_{6}\right)$ are standard. In Theorems 2 and 3 we prove that if $n \geqq 7$, then Aut $F\left(Z_{n}\right)$ has only standard automorphisms and hence, Aut $F\left(Z_{n}\right)$ is isomorphic to Aut $Z_{n}$. If $Q$ denotes the additive group of rationals and $G$ is a subgroup of $Q / Z$, we can use the preceding results to characterize Aut $F(G)$ in terms of Aut $G$. It appears that the absence of retractions (retractable groups are torsion-free) might restrict the number of nonstandard 
automorphisms. In Theorem 4 we show that an automorphism of $F(G)$ is standard if and only if the automorphism is inclusion preserving.

In $\S 2$ (Theorem 1) we show that if $A \in F\left(Z_{n}\right)$ and $\theta \in \operatorname{Aut} F\left(Z_{n}\right)$, then $|A|=|A \theta|$. This theorem was crucial for our work. Our results are computational in nature and are established through a sequence of lemmas. If $X$ and $Y$ are sets, then $X \backslash Y$ denotes the set of elements in $X$ but not in $Y$.

2. Preliminaries. We have been unable to determine if the elements of Aut $F(G)$ preserve the cardinality of subsets of $G$. The purpose of this section is to prove that the elements of Aut $F\left(Z_{n}\right)$ do preserve set cardinality.

Lemma 1. Let $G$ be a finite group and let $A, B \in F(G)$ with $|A|<|B|<|G|$. Then there exists $g \in G \backslash\{0\}$ such that $|A+\{0, g\}|<$ $|B+\{0, g\}|$ and $|B|<|B+\{0, g\}|$.

Proof. To prove the lemma it suffices to take $|B|=|A|+1$. If $G \backslash A=\left\{x_{1}, \cdots, x_{t+1}\right\}$ and $G \backslash B=\left\{y_{1}, \cdots, y_{t}\right\}$, let

$$
A^{*}=\left\{\left(\left(x_{i}, x_{j}\right), g_{i j}\right) \mid i, j \in\{1, \cdots, t+1\}, i \neq j \text {, and }-x_{i}+x_{j}=g_{i j}\right\}
$$

and

$$
B^{*}=\left\{\left(\left(y_{r}, y_{s}\right), g_{r s}\right) \mid r, s \in\{1, \cdots, t\}, r \neq s \text {, and }-y_{r}+y_{s}=g_{r s}\right\} .
$$

Then $\left|A^{*}\right|=t(t+1)>t(t-1)=\left|B^{*}\right|$ and $0 \notin A^{*} \pi_{2} \cup B^{*} \pi_{2}$.

Case 1. There exists $g \in A^{*} \pi_{2} \backslash B^{*} \pi_{2}$. Then $g=-x_{i}+x_{j}$ for some $i \neq j$. Since $x_{i} \notin A, x_{j}=x_{i}+g \notin A+g$ and since $x_{j} \notin A, x_{j} \notin A \cup$ $(A+g)=A+\{0, g\}$. Thus $|A+\{0, g\}|<|G|$. If $y \in G \backslash B$, then there exists $z \in G$ such that $z+g=y$, and $g \neq 0$ implies that $z \neq y$. Since $g \notin B^{*} \pi_{2}$, we have that $z \notin G \backslash B$. Hence $z \in B$ and so $y \in B+g$. Therefore we have that

$$
|A+\{0, g\}|<|G|=|B+\{0, g\}| \text { and }|B|<|B+\{0, g\}| \text {. }
$$

Case 2. $A^{*} \pi_{2} \subseteq B^{*} \pi_{2}$. Since $\left|A^{*}\right|>\left|B^{*}\right|$, there exists $g \in A^{*} \pi_{2}$ such that if

$$
J=\left\{\left(x_{i}, x_{j}\right) \mid\left(\left(x_{\imath}, x_{j}\right), g\right) \in A^{*}\right\}
$$

and

$$
K=\left\{\left(y_{r}, y_{s}\right) \mid\left(\left(y_{r}, y_{s}\right), g\right) \in B^{*}\right\},
$$

then $|J|>|K|$. Let $J=\left\{\left(x_{i_{1}}, x_{j_{1}}\right), \cdots,\left(x_{i_{m}}, x_{j_{m}}\right)\right\}$ and $K=\left\{\left(y_{r_{1}}, y_{s_{1}}\right)\right.$, 
$\left.\cdots,\left(y_{r_{n}}, y_{s_{n}}\right)\right\}$, where $m>n$. If $1 \leqq k \leqq m$, then $x_{j_{k}}=x_{i_{k}}+g$ and since $x_{i_{k}}, x_{j_{k}} \notin A, x_{j_{k}} \notin A+\{0, g\}$. If $x \in G \backslash\left(A \cup\left\{x_{j_{1}}, \cdots, x_{j_{m}}\right\}\right)$, then $x=z+g$ for some $z \in G, z \neq x$. Since $x \notin J \pi_{2}$, we have that $z \in A$. Thus $x \in A+\{0, g\}$. Consequently, $A+\{0, g\}=G \backslash\left\{x_{j_{1}}, \cdots, x_{j_{m}}\right\}$. Similarly, $B+\{0, g\}=G \mid\left\{y_{s_{1}}, \cdots, y_{s_{n}}\right\}$ and so $|A+\{0, g\}|<|B+\{0, g\}|$.

Suppose (by way of contradiction) that $|B|=|B+\{0, g\}|$. Then $B=B+\{0, g\}=G \backslash\left\{y_{s_{1}}, \cdots, y_{s_{n}}\right\}$ and hence $n=t$ and $m=t+1$. Thus for each $x_{i}, x_{i}+g=x_{j}$ for some $x_{j}$. Then $x_{i}+2 g=x_{j}+g=x_{k}$, for some $k, 1 \leqq k \leqq t+1$. It follows, by induction, that $x_{i}+\langle g\rangle \leqq$ $G \backslash A$ for each $i, 1 \leqq i \leqq t+1$, and so $G \backslash A$ is a union of cosets of $\langle g\rangle$. Therefore, $o(g) \mid(t+1)$. Similarly, $o(g) \mid t$ and this is a contradiction as $g \neq 0$. Hence, $|B|<|B+\{0, g\}|$.

If $A$ is an element of the semigroup $F(G)$, define $1 A=A$ and for $n>1$, define $n A=(n-1) A+A$. Note that $n A$ does not necessarily equal $\{n a \mid a \in A\}$.

Lemma 2. Let $G$ be a finite abelian group. If $A \in F(G)$ and $|A+k A|=|k A|$ for some $k \geqq 1$, then $|l A+k A|=|k A|$ for all $l \geqq 1$.

Proof. The equality holds for $l=1$. Assume that $l \geqq 1$ and that $|l A+k A|=|k A|$. If $a \in l A$, then $|l A+k A|=|-a+l A+k A|$ and since $k A \subseteq-\alpha+l A+k A$, we have that $k A=-\alpha+l A+k A$. Therefore, $|k A|=|A+k A|=|A+(-a+l A+k A)|=|(l+1) A+k A|$. By induction, the lemma holds for all $l \geqq 1$.

The proof of the next lemma is straightforward and will be omitted.

LEMMA 3. Let $G$ be a group and let $H$ be a finite subgroup of G. If $\theta \in$ Aut $F(G)$, then

(i ) $\theta \mid F(H)$ is a semigroup isomorphism of $F(H)$ onto $F(H \theta)$;

(ii) $H$ and $H \theta$ are isomorphic subgroups of $G$;

(iii) if $\theta \mid G$ is the identity, then $H=H \theta$.

LEMMA 4. If $G$ is a finite group, $b \in G \backslash\{0\}$, and $\theta \in$ Aut $F(G)$, then $|\{0, b\} \theta|=2$.

Proof. Let $\theta \in$ Aut $F(G)$ and $\eta$ be the standard automorphism of $F(G)$ induced by $(\theta \mid G)^{-1}$. Since $\eta$ preserves set cardinality, $|A \theta \eta|=|A \theta|$ for all $A \in F(G)$, and $\theta \eta \mid G=\iota$ where $\iota$ denotes the identity mapping of $G$. Thus we may assume that $\theta \mid G=\iota$. If $n=o(b)$ and $H=\langle b\rangle$, then $H=(n-1)\{0, b\}$. By Lemma $3, H=H \theta=$ $(n-1)(\{0, b\} \theta)$. Further, $n-1$ is the smallest natural number such 
that $(n-1)(\{0, b\} \theta)=H$. If $A=\{0, b\} \theta$, then $A \subseteq H$ and $(n-1) A=H$. If $B \subseteq G,|B|>2$, and $k \geqq 1$, then by induction and Lemma 2, $|(k+1) B|>k+2$ or $|(k+1) B|=|k B|$. In particular, if $|A|>2$, then since $|((n-2)+1) A|=n$, we must have that $n=|(n-1) A|=$ $|(n-2) A|$. Thus $(n-2) A=H$, and this is a contradiction.

Corollary 1. Let $G$ be a finite group and $a, b \in G$ with $a \neq b$. If $\theta \in$ Aut $F(G)$, then $|\{a, b\} \theta|=2$.

LemMa 5. If $G$ is a finite group and $A \in F(G)$ with $|A|=$ $|G|-1$, then $|A \theta|=|A|$ for all $\theta \in$ Aut $F(G)$.

Proof. As in Lemma 4, we may assume $\theta \mid G=\iota$. Let $a, b \in G$ with $a \neq b$. Since $|\{a, b\}|+|A|=|G|+1,\{a, b\}+A=G[3$, Theorem 1]. Suppose (by way of contradiction) that $A \theta=B$, where $|B|<|A|$. Then there exists $x, y \in G \backslash B$ with $x \neq y$. Since $x \notin\{0, x-y\}+B$, $\{0, x-y\}+B \neq G$. By the preceding corollary, $\{0, x-y\}=\{a, b\} \theta$ for some $a, b \in G$ with $a \neq b$. But then

$$
\{a, b\}+A=G=G \theta=(\{a, b\}+A) \theta=\{0, x-y\}+B \neq G,
$$

a contradiction. Hence, $|A \theta|=|A|$.

If $A, B \in F(G)$ and $A=g+B$ for some $g \in G$, then $A$ is said to be a (left) translate of $B$. Clearly $F(G)$ is the union of mutually disjoint translation classes. Moreover, if $G$ is abelian and $\theta \in \operatorname{Aut} F(G)$, then $\theta$ is completely determined by it action on the group of units of $F(G)$ and a system of representatives for the translation classes.

Lemma 6. Let $\theta \in$ Aut $Z_{n}$ and $\{0,1\} \theta=g+\{0,1\}$. If $1 \leqq a \leqq$ $n-1$, then there exists $h \in Z_{n}$ such that $\{0, a\} \theta=h+\{0, a\}$.

Proof. The translation class of $\{0, a\}$ is the same as the translation class of $\{0, n-a\}$. Thus we may assume that $a \leqq n / 2$. If $n$ is even and $a=n / 2$, then $\{0, a\}$ is the only subgroup of $Z_{n}$ of order 2. By Lemma $3,\{0, a\} \theta=\{0, a\}$. Therefore, we may further assume that $1 \leqq a<n / 2$. By induction we may assume that we have verified the lemma for all $b$ such that $1 \leqq b<a<n / 2$. If $\{0, a\} \theta=$ $y+\{0, t\}$, then, since $\{0, t\}=t+\{0, n-t\}$, we may assume $t \leqq n / 2$ and since $\theta$ maps a translation class onto a translation class, $a \leqq t$. Now we have the equation $(a-1)\{0,1\}+\{0, a\}=(2 a-1)\{0,1\}$ and, taking the image of both sides under $\theta$, we obtain

$$
(a-1) g+(a-1)\{0,1\}+y+\{0, t\}=(2 a-1) g+(2 a-1)\{0,1\} .
$$

Hence, $\{0,1, \cdots, a-1, t, t+1, \cdots, t+a-1\}$ is a translate of 
$\{0,1, \cdots, 2 a-1\}$. Since $t+a-1<n-1, t=a$ is the only possible solution.

THEOREM 1. If $A \in F\left(Z_{n}\right)$ and $\theta \in$ Aut $F\left(Z_{n}\right)$, then $|A \theta|=|A|$.

Proof. Let $\{0,1\} \theta=g+\{0, b\}$. Since $(n-1)\{0,1\}=Z_{n}$, it follows that $b$ is a generator for $Z_{n}$. Let $\Psi$ be the standard automorphism of $F\left(Z_{n}\right)$ induced by the automorphism of $Z_{n}$ that maps $b$ to 1 . Now $\Psi$ preserves cardinality and hence, $\theta \Psi$ will preserve cardinality if and only if $\theta$ preserves cardinality. Thus we may assume that $\{0,1\} \theta=g+\{0,1\}$. Suppose (by way of contradiction) that there exists $B \in F\left(Z_{n}\right)$ such that $|B \theta| \neq|B|$. We may assume that if $A \in F(G)$ with $|A|>|B|$, then $|A \theta|=|A|$. Thus, $|B \theta|<|B|$ and by Lemma $5,|B|<n-1$. By Lemma 1 , there exists $x \in Z_{n}$ such that $|B|<|B+\{0, x\}|$ and $|B \theta+\{0, x\}|<|B+\{0, x\}|$, and by Lemma $6,\{0, x\} \theta=h+\{0, x\}$, for some $h$. Therefore,

$$
\begin{aligned}
|(B+\{0, x\}) \theta| & =|B \theta+h+\{0, x\}| \\
& =|B \theta+\{0, x\}|<|B+\{0, x\}| \\
& =|(B+\{0, x\}) \theta| .
\end{aligned}
$$

This is a contradiction and hence, $|A \theta|=A$ for all $A \in F\left(Z_{n}\right)$.

3. Determination of Aut $F\left(Z_{n}\right)$. Let $G$ be a group, $H$ be the group of standard automorphisms of $F(G)$, and $K$ be the group of automorphisms of $F(G)$ that are the identity on the group of units of $F(G)$. Then $K$ is a normal subgroup of Aut $F(G), H \cap K=\{c\}$, and Aut $F(G)=K H$. If $\theta \in K$ and $G$ is abelian, then $\theta$ is uniquely determined by its action on a system of representatives of the translation classes of $F(G)$. Clearly $F\left(Z_{2}\right)$ admits only standard automorphisms. The verification of the following assertions are computational (some are lengthy) and will be omitted. If $G=Z_{3}$, then there exists $\theta \in$ Aut $F(G)$ with $\theta \mid G=\iota,\{0,1\} \theta=\{0,2\},\{0,1,2\} \theta:=\{0,1,2\}$; $K=\left\{\iota, \theta, \theta^{2}\right\}, \quad H=\{\ell, \beta\}$ where $(1) \beta=2$, and $\beta^{-1} \theta \beta=\theta^{2}$. Thus, Aut $F(G)=K H$ and is isomorphic to $S_{3}$, the symmetric group of degree 3. If $G=Z_{4}$, then there exists $\theta \in \operatorname{Aut} F(G)$ with $\theta \mid G=\iota$, $\{0,1\} \theta=\{0,3\},\{0,2\} \theta=\{0,2\},\{0,1,2\} \theta=\{0,2,3\},\{0,1,2,3\} \theta=\{0,1,2,3\}$; $K=\left\{\iota, \theta, \theta^{2}, \theta^{3}\right\}, H=\{\iota, \beta\}$, where $(1) \beta=3$, and $\beta^{-1} \theta \beta=\theta^{3}$. Thus, Aut $F(G)=K H$ and is isomorphic to $D_{4}$, the dihedral group of order 8. If $G=Z_{5}$, then there exists $\theta \in F(G)$ with $\theta \mid G=\iota,\{0,1\} \theta=\{2,4\}$, $\{0,2\} \theta=\{3,4\},\{0,1,2\} \theta=\{1,3,4\},\{0,1,3\} \theta=\{2,3,4\},\{0,1,2,3\} \theta=$ $\{0,1,2,3\}, \quad\{0,1,2,3,4\} \theta=\{0,1,2,3,4\} ; K=\{\iota, \theta\}, \quad H=\left\{\iota, \eta, \eta^{2}, \eta^{3}\right\}$, where (1) $\eta=2$, and $\theta \eta=\eta \theta$. Thus, Aut $F(G)=K H$ and is isomorphic to the direct product of $Z_{2}$ and $Z_{4}$. Finally, if $G=Z_{6}$, then $K=\{c\}$ 
and Aut $F(G)$ is isomorphic to Aut $G$.

The remaining portion of this paper is devoted to showing that Aut $F(G)$ consists only of standard automorphisms if $G$ is a subgroup of $Q / Z$ and $|G| \geqq 7$, and hence Aut $F(G)$ is isomorphic to Aut $G$. The proofs of the next three lemmas are straightforward and will be omitted.

Lemma 7. If $A \in F(G)$ and $L(A)=\{g \mid g+A=A\}$, then $L(A)$ is a subgroup of $G$ and $A$ is a union of right cosets of $L(A)$. If $G$ is finite, then the number of translates of $A$ is the index of $L(A)$ in $G$.

LemMa 8. If $G$ is a finite group, $A \in F(G)$, and $|A|=|G|-1$, then $L(A)=\{0\}$ and all subsets of $G$ of cardinality $|G|-1$ belong to the translation class of $A$.

Lemma 9. If $a$ is a generator of $Z_{n}$ and $A=k\{0, a\}$, where $1<k \leqq n-2$, then $L(A)=\{0\}$.

For the remainder of this paper we shall assume that $n \geqq 7$.

Lemma 10. If $\theta \in$ Aut $F\left(Z_{n}\right)$ and $\{0,1\} \theta=\{0,1\}$, then $\{0, r\} \theta=$ $\{0, r\}$ for every $r \in Z_{n} \mid\{0\}$ and $\theta \mid Z_{n}$ is the identity.

Proof. We first assume that $1<r \leqq n / 2$. If $r=n / 2$, then $\{0, r\}$ is a subgroup of $Z_{n}$ and by Lemma $3,\{0, r\} \theta=\{0, r\}$ since it is the only subgroup of order two. Thus we may suppose that $1<r<n / 2$. By Lemma 6, $\{0, r\} \theta=h+\{0, r\}$ for some $h \in Z_{n}$. Now

$$
(r-1)\{0,1\}+\{0, r\}=(2 r-1)\{0,1\} .
$$

If we apply $\theta$ to each side of this equation, we have that

$$
(r-1)\{0,1\}+h+\{0, r\}=(2 r-1)\{0,1\} .
$$

It follows from Lemma 9 that $h=0$ and so $\{0, r\} \theta=\{0, r\}$.

We now show that $(1) \theta=1$. We do this by considering separately the cases where $n$ is even and $n$ is odd.

Case 1. $n$ is even. Then $\{0,1\}+\{0,1,3\}=\{0,1,2,3,4\}=4\{0,1\}$. Applying $\theta$ to this equation we have $\{0,1\}+\{a, b, c\}=4\{0,1\}$. It follows that $\{a, b, c\}=\{0,1,3\}$ or $\{a, b, c\}=\{0,2,3\}$. Now the following equalities hold:

$$
\frac{n-4}{2}\{0,2\}+\{0,1,3\}=(n-1)+(n-2)\{0,1\}
$$


and

$$
\frac{n-4}{2}\{0,2\}+\{0,2,3\}=2+(n-2)\{0,1\} \text {. }
$$

Suppose that $\{0,1,3\} \theta=\{0,2,3\}$. Then, using (1), we obtain

$$
\frac{n-4}{2}\{0,2\}+\{0,2,3\}=(n-1) \theta+(n-2)\{0,1\} \text {. }
$$

Using this equation, equation (2), and Lemma 9, we have that $(n-1) \theta=2$ which is a contradiction since 2 is not a generator of $Z_{n}$. Thus $\{0,1,3\} \theta=\{0,1,3\}$. Applying $\theta$ to equation (1) and by Lemma 9 , we have $(n-1) \theta=n-1$ and hence, (1) $\theta=1$.

Case 2. $n$ is odd. In this case we have the equation

$$
1+(n-2)\{0,2\}=(n-2)\{0,1\} \text {. }
$$

By applying $\theta$ to this equation we conclude that $(1) \theta=1$.

Next suppose that $n / 2<r \leqq n$. Then $\{0, r\}=r+\{0, n-r\}$, so that $\{0, r\} \theta=r \theta+\{0, n-r\} \theta=r+\{0, n-r\}=\{0, r\}$.

Lemma 11. If $\theta \in \operatorname{Aut} F\left(Z_{n}\right)$ and $\{0,1\} \theta=\{0,1\}$, then $A \theta=A$ for every $A \in F\left(Z_{n}\right)$.

Proof. By the preceding lemma, $\{0, r\} \theta=\{0, r\}$ for every $r \epsilon$ $Z_{n} \mid\{0\}$ and $\theta \mid Z_{n}$ is the identity. If $A \in F\left(Z_{n}\right)$ and $|A|=n-1$, then $A$ is a translate of $(n-2)\{0,1\}$ and so $A \theta=A$. Suppose (by way of contradiction) that there exists $A \in F\left(Z_{n}\right)$ such that $A \theta \neq A$. Then $|A| \leqq n-2$ and we may assume that if $B \in F(G)$ with $|A|<|B|$, then $B \theta=B$. Let $w \in A \theta \backslash A$ and $u \in Z_{n} \backslash A$ with $w \neq u$. Then $w \notin A+\{0, w-u\}$, but $w \in A \theta+\{0, w-u\}=(A+\{0, w-u\}) \theta$. By the maximalily of $|A|,|A| \geqq|A+\{0, w-u\}| \geqq|A|$ and so $A=A+$ $\{0, w-u\}$. Therefore, $w-u \in L(A)$ and so $|L(A)| \geqq 2$. By Lemma $7, A=\bigcup_{i=1}^{t}\left(L(A)+a_{i}\right)$. Since subgroups of $Z_{n}$ are fixed by $\theta$, all cosets of $L(A)$ are fixed by $\theta$. Hence, $t \geqq 2$. Now $L(A)+u \nsubseteq A$. Since we have shown that $w-u \in L(A)$ for every $u \in Z_{n} \backslash A$, we have that $Z_{n} \backslash A$ is a single coset of $L(A)$ and hence, $A$ is the union of all but one coset of $L(A)$. Let $a$ be the smallest positive integer such that $L(A)=\langle a\rangle$. Then $a\rangle 2$ and a system of representatives for the cosets of $L(A)$ in $Z_{n}$ is $\{0,1, \cdots, a-1\}$. We may assume that $\left\{a_{1}, \cdots, a_{t}\right\} \subseteq\{0,1, \cdots, a-1\}$. Let $b \in\{0,1, \cdots, a-1\}$ such that $L(A)+b=L(A)+w$. If $x=(a-1)-b$, then $\left\{a_{1}+x, \cdots, a_{t}+x\right\}$ is a system of representatives for all but one coset of $L(A)$. The 
coset not included is $L(A)+(a-1)$. Then $L(A)+\{0,1, \cdots, a-2\}=$ $\bigcup_{i=1}^{t}\left(L(A)+a_{i}+x\right)=A+x$. Now $L(A) \theta=L(A)$ and

$$
\{0,1, \cdots, a-2\} \theta=(a-2)\{0,1\}
$$

and consequently, $(A+x) \theta=A+x$. Since $x \theta=x$, we have that $A \theta=A$, which is a contradiction. Thus the lemma is proven.

LEMMA 12. If $\theta \in \operatorname{Aut} F\left(Z_{n}\right)$ and $\{0,1\} \theta=c+\{0,1\}$ for some $c \in Z_{n}$, then $\{0, a\} \theta=a c+\{0, a\}$ for $1 \leqq a<n / 2$.

Proof. By Lemma 6, $\{0, a\} \theta=h+\{0, a\}$ for some $h \in Z_{n}$. If $2 \leqq a<n / 2$, then $(a-1)\{0,1\}+\{0, a\}=(2 a-1)\{0,1\}$. If we apply $\theta$ to this equation we obtain $(a-1) c+(a-1)\{0,1\}+h+\{0, a\}=$ $(2 a-1) c+(2 a-1)\{0,1\}$. Thus, $(a-1) c+h \equiv(2 a-1) c($ modulo $n)$, and so $h \equiv a c(\operatorname{modulo} n)$.

LEMMA 13. Let $a \in Z_{n}$ with $2 \leqq a \leqq n / 2$. Then

(i) if $k$ and $l$ are positive integers such that $k\{0,1\}+\{0,1, a\}=$ $l\{0,1\}$, then $a-2 \leqq k$ and $2 a-2 \leqq l$;

(ii) if $k$ and $l$ are positive integers such that $k\{0,1\}+$ $\{0, a-1, a\}=l\{0,1\}$, then $a-2 \leqq k$ and $2 a-2 \leqq l$;

(iii) if $1<b<a-1$, then there exist positive integers $k$ and $l$ such that $k<a-2$ and $k\{0,1\}+\{0, b, a\}=l\{0,1\}$;

(iv) if $x, y, z \in Z_{n}$ with $x<y<z<n$ and $(a-2)\{0,1\}+$ $\{x, y, z\}=(2 a-2)\{0,1\}$, then $x=0$ and $z=a$;

(v) $(a-2)\{0,1\}+\{0,1, a\}=(2 a-2)\{0,1\}$;

(vi) $(a-2)\{0,1\}+\{0, a-1, a\}=(2 a-2)\{0,1\}$.

Proof. Clearly (v) and (vi) hold. To see that (i) is true, we observe that if $1 \leqq k<a-2$, then $a-1 \notin k\{0,1\}+\{0,1, a\}$ and so there is no natural number $l$ such that $k\{0,1\}+\{0,1, a\}=l\{0,1\}$. Thus if $k$ and $l$ are natural numbers such that $k\{0,1\}+\{0,1, \alpha\}=$ $l\{0,1\}$, then $a-2 \leqq k$ and so $2 a-2 \leqq l$. The proof of (ii) is similar and will be omitted. For (iii), let $1<b<a-1$ and $k=\max \{b-1$, $a-(b+1)\}$. If $k=b-1$, then $k\{0,1\}+\{0, b, a\}=(a+b-1)\{0,1\}$ and $k=b-1<a-2$. If $k=a-(b+1)$, then $k\{0,1\}+\{0, b, a\}=$ $(2 a-b-1)\{0,1\}$ and $k=a-(b+1)<a-2$. For (iv) we suppose that $x<y<z$ and $(a-2)\{0,1\}+\{x, y, z\}=(2 a-2)\{0,1\}$. Then we have $\{x, x+1, \cdots, x+a-2\} \cup\{y, y+1, \cdots, y+a-2\} \cup\{z, z+$ $1, \cdots, z+a-2\}=\{0,1, \cdots, 2 a-2\}$. The elements from $2 a-1$ to $n-1$ belong to $Z_{n}$ but not to the right hand side. The left hand side is the union of three consecutive listings and so the elements from $2 a-1$ to $n-1$ must occur between $x+a-2$ and $y, y+a-2$ 
and $z$, or $z+a-2$ and $x$. The first two cases cannot occur as this would force $y$ or $z$ to be larger than $n-1$. Thus, $z+a-2=2 a-2$ and so $z=a$. Then $x=0$.

LEMma 14. Let $a \in Z_{n}$ with $1<a \leqq n / 2$ and $\theta \in$ Aut $F\left(Z_{n}\right)$ such that $\{0,1\} \theta=c+\{0,1\}$. Then

(i) $\{0,1, a\} \theta$ is in the translation class of either $\{0,1, a\}$ or $\{0, a-1, a\}$

(ii) $\{0, a-1, a\} \theta$ is in the translation class of either $\{0,1, a\}$ or $\{0, a-1, a\}$;

(iii) if $\{0,1, a\} \theta$ is in the translation class of $\{0,1, a\}$, then $\{0,1, a\} \theta=a c+\{0,1, a\}$ and $\{0, a-1, a\} \theta=a c+\{0, a-1, a\}$

(iv) if $\{0,1, a\} \theta$ is in the translation class of $\{0, a-1, a\}$, then $\{0,1, a\} \theta=a c+\{0, a-1, a\}$ and $\{0, a-1, a\} \theta=a c+\{0,1, a\}$.

Proof. (i) Let $\{0,1, a\} \theta=\{x, y, z\}$. By (v) of Lemma 13, $(a-2)\{0,1\}+\{0,1, a\}=(2 a-2)\{0,1\}$. If we apply $\theta$ to this equation we obtain the equation

$$
(a-2) c+(a-2)\{0,1\}+\{x, y, z\}=(2 a-2) c+(2 a-2)\{0,1\} .
$$

Thus

$$
(a-2)\{0,1\}+\{x-a c, y-a c, z-a c\}=(2 a-2)\{0,1\} .
$$

Without loss of generality we may assume that $0 \leqq x-a c<y-$ $a c<z-a c<n$. By Lemma 13 (iv), $x-a c=0$ and $z-a c=a$. Let $b=y-a c$. Suppose (by way of contradiction) that $1<b<a-1$. Then by Lemma 13 (iii), there are positive integers $k$ and $l$ such that $k<a-2$ and $k\{0,1\}+\{x-a c, y-a c, z-a c\}=l\{0,1\}$. Thus

$$
(a-2) c+k\{0,1\}+\{x, y, z\}=(2 a-2) c+l\{0,1\} .
$$

Applying $\theta^{-1}$ to this last equation, we obtain an equation of the form

$$
d+k\{0,1\}+\{0,1, a\}=f+l\{0,1\} .
$$

Hence,

$$
\begin{aligned}
& ((a-2)-k)\{0,1\}+k\{0,1\}+\{0,1, a\} \\
& \quad=(f-d)+((a-2)-k+l)\{0,1\}
\end{aligned}
$$

and so

$$
(a-2)\{0,1\}+\{0,1, a\}=(f-d)+((a-2)-k+l)\{0,1\} .
$$

Hence, $(2 a-2)\{0,1\}=(f-d)+((a-2)-k+l)\{0,1\}$. It follows 
that $f \equiv d$ (modulo $n$ ). Therefore, $k\{0,1\}+\{0,1, a\}=l\{0,1\}$, but this contradicts Lemma 13 (i). Consequently, $\{0,1, a\} \theta$ is in the class of $\{0,1, a\}$ or $\{0, a-1, a\}$.

The proof of (ii) is similar. Parts (iii) and (iv) then follow from what has been shown.

The proof of the next lemma is straightforward and will be omitted.

LEMMA 15. If $n$ is even, $a \in Z_{n}, a$ is odd, and $1<a \leqq n / 2$, then

(i) $(n-4) / 2\{0,2\}+\{0,1, a\}=(n-1)+(n-2)\{0,1\}$;

(ii) $(n-4) / 2\{0,2\}+\{0, a-1, a\}=(a-1)+(n-2)\{0,1\}$.

LEMMA 16. If $n$ is even, $\theta \in \operatorname{Aut} F\left(Z_{n}\right)$, and $\{0,1\} \theta=c+\{0,1\}$, then $\theta$ is a standard automorphism and $c=0$ or $c=n-1$.

Proof. By Lemma 15,

$$
1+\frac{n-4}{2}\{0,2\}+\{0,1,3\}=(n-2)\{0,1\} .
$$

By Lemma 14, $\{0,1,3\} \theta=3 c+\{0,1,3\}$ or $\{0,1,3\} \theta=3 c+\{0,2,3\}$.

Case 1. $\{0,1,3\} \theta=3 c+\{0,1,3\}$. Then $\{0,2,3\} \theta=3 c+\{0,2,3\}$. If we apply $\theta$ to equation (1), then by Lemma 12, we obtain that

$$
\text { (1) } \begin{aligned}
& +\frac{n-4}{2}(2 c)+\frac{n-4}{2}\{0,2\}+3 c+\{0,1,3\} \\
& =(n-2) c+(n-2)\{0,1\}
\end{aligned}
$$

so that

$$
\text { (1) } \theta+c+\frac{n-4}{2}\{0,2\}+\{0,1,3\}=(n-2)\{0,1\} \text {. }
$$

By equation (1), we have that $(1) \theta+c+(n-1)+(n-2)\{0,1\}=$ $(n-2)\{0,1\}$ and hence, $(1) \theta+c+n-1 \equiv 0$ (modulo $n$ ). Consequently, (1) $\theta+c-1 \equiv 0$ (modulo $n$ ). By Lemma 15, we have that

$$
\frac{n-4}{2}\{0,2\}+\{0,2,3\}=2+(n-2)\{0,1\} \text {. }
$$

Applying $\theta$ to this equation we obtain that $-2(1) \theta+c+2 \equiv 0$ (modulo $n$ ). Thus, $3 c \equiv 0$ (modulo $n$ ). If $n=8$, then $c=0$ and by Lemma $11, \theta=\iota$. Suppose that $n \geqq 10$. Then by Lemma 15, we have that 


$$
1+\frac{n-4}{2}\{0,2\}+\{0,1,5\}=(n-2)\{0,1\}
$$

and

$$
\frac{n-4}{2}\{0,2\}+\{0,4,5\}=4+(n-2)\{0,1\} .
$$

Subcase 1.1. $\{0,1,5\} \theta=5 c+\{0,1,5\}$. Then we have that

$$
\begin{aligned}
(1) \theta & +(n-4) c+\frac{n-4}{2}\{0,2\}+5 c+\{0,1,5\} \\
& =(n-2) c+(n-2)\{0,1\} .
\end{aligned}
$$

Hence, $(1) \theta+3 c-1+(n-2)\{0,1\}=(n-2)\{0,1\}$ and so $(1) \theta+3 c-$ $1 \equiv 0(\operatorname{modulo} n)$. Since $3 c \equiv 0(\operatorname{modulo} n)$, we have that $(1) \theta=1$ and $c=0$. Therefore, by Lemma $11, \theta=\imath$.

Subcase 1.2. $\quad\{0,1,5\} \theta=5 c+\{0,4,5\} . \quad$ By an argument similar to the one given in Subcase 1.1, we obtain that $(1) \theta+3 c+4 \equiv 0$ $(\operatorname{modulo} n)$. Since $3 c \equiv 0(\operatorname{modulo} n),(1) \theta \equiv(n-4)$ (modulo $n)$, but this is impossible as $n-4$ is not a generator of $Z_{n}$.

Case 2. $\{0,1,3\} \theta=3 c+\{0,2,3\}$. Then by Lemma 15 and the same techniques as above, we obtain the congruences $(1) \theta+c+2 \equiv 0$ $(\operatorname{modulo} n)$ and $c-2(1) \theta-1 \equiv 0 \quad(\operatorname{modulo} n)$. Then $3 c \equiv(n-3)$ (modulo $n$ ). If $n=8$, then $c \equiv 7$ (modulo 8 ) and $(1) \theta=7$. If $\eta$ is the standard automorphism of $F\left(Z_{n}\right)$ that takes 1 to -1 , then $\{0,1\} \theta \eta=\{0,1\}$ and so, by Lemma $11, \theta \eta=c$. Thus, $\theta=\eta^{-1}$ and hence $\theta$ is standard. If $n \geqq 10$, then, as in Case 1 , (1) $\theta=n-1$ and $c=n-1$. Thus, $\theta$ is standard.

THEOREM 2. If $n$ is even, then Aut $F\left(Z_{n}\right)$ consists only of standard automorphisms and so is isomorphic to Aut $Z_{n}$.

Proof. If $\theta \in \operatorname{Aut} F\left(Z_{n}\right)$, then $\{0,1\} \theta=h+\{0, r\}$ for some $h, r \in Z_{n}$. Let $\eta$ be the standard automorphism of $F\left(Z_{n}\right)$ that takes $r$ to 1 . Then $\{0,1\} \theta \eta=c+\{0,1\}$ for some $c \in Z_{n}$. By Lemma 16, $\theta \eta=\Psi$ is a standard automorphism and hence $\theta=\psi \eta^{-1}$ is standard.

For $n$ odd, we proceed almost as above.

Lemma 17. If $n$ is odd, then

(i) $(n-3) / 2\{0,2\}+\{0,1,3\}=(n-2)\{0,1\}$;

(ii) $(n-3) / 2\{0,2\}+\{0,2,3\}=2+(n-2)\{0,1\}$. 
THEOREM 3. If $n$ is odd, then Aut $F\left(Z_{n}\right)$ consists only of standard automorphisms and so is isomorphic to Aut $Z_{n}$.

Proof. If $\theta \in$ Aut $F\left(Z_{n}\right)$, then $\{0,1\} \theta=h+\{0, r\}$ for some $h, r \in Z_{n}$. Let $\eta$ be the standard automorphism of $F\left(Z_{n}\right)$ that takes $r$ to 1. Then $\{0,1\} \theta \eta=c+\{0,1\}$ for some $c \in Z_{n}$. If $\{0,1,3\} \theta \eta=3 c+$ $\{0,1,3\}$, then we apply $\theta \eta$ to (i) of Lemma 17 and obtain the congruence $2 c \equiv 0$ (modulo $n$ ) and so $c \equiv 0$ (modulo $n$ ). Thus, by Lemma $11, \theta \eta$ is a standard automorphism and consequently, $\theta$ is standard. The same conclusion holds if $\{0,1,3\} \theta \eta=3 c+\{0,2,3\}$.

The following theorem gives a characterization of standard automorphisms for arbitrary groups. It was proven in [1, Theorem 5] and, for completeness, we repeat the proof here.

THeOREM 4. If $G$ is a group and $\theta \in$ Aut $F(G)$, then $\theta$ is a standard automorphism if and only if $A, B \in F(G)$ with $A \subseteq B$ implies that $A \theta \subseteq B \theta$.

Proof. Clearly if $\theta$ is a standard automorphism, then $\theta$ preserves set containment. Conversely suppose that $\theta$ is inclusion preserving, let $\alpha=\theta \mid G$, and $\theta_{\alpha}$ be the standard automorphism of $F(G)$ induced by $\alpha$. We proceed by induction on the cardinality of the sets in $F(G)$. If $A \in F(G)$ such that $|A|=1$, then $A \theta=A \theta_{\alpha}$. Assume that for all $A \in F(G)$ with $|A| \leqq k, A \theta=A \theta_{\alpha}$, and let $B \in F(G)$ with $|B|=k+1$. If $D=B \theta_{\alpha}$, then there exists $C \in F(G)$ such that $C \theta=D$. Since $\theta$ is inclusion preserving, if $b \in B$, then $b \alpha=b \theta \in B \theta$. Hence, $B \theta_{\alpha} \subseteq B \theta$. If $x \in C$, then $x \alpha \in C \theta=D$. Thus, $x \alpha=b \alpha$ for some $b \in B$ and so $x=b$. Therefore, $C \subseteq B$. If $C \neq B$, then, by the inductive hypothesis, $C \theta=C \theta_{\alpha}=D=B \theta_{\alpha}$ and so $C=B$. Therefore, $C=B$ and so $B \theta=$ $C \theta=D=B \theta_{\alpha}$. Thus, $\theta$ is the standard automorphism $\theta_{\alpha}$.

We now extend our results to a larger class of groups.

THEOREM 5. If $G$ is a subgroup of $Q / Z$ such that $|G|>5$, then Aut $F(G)$ consists only of standard automorphisms and hence Aut $F(G)$ is isomorphic to Aut $G$.

Proof. If $G$ is finite, then $G$ is cyclic with $|G|>5$ and so Aut $F(G)$ consists only of standard automorphisms. Suppose that $G$ is infinite and let $A, B \in F(G)$ with $A \subseteq B$, and let $\theta \in$ Aut $F(G)$. Then there is a finite cyclic subgroup $H$ of $G$ such that $B \subseteq H$ and $|H|>5$. Since $H$ is the only subgroup of $G$ of order $|H|$, we have, by Lemma $3, H=H \theta$. Thus, $\theta \mid F(H) \in \operatorname{Aut} F(H)$ and so $\theta \mid F(H)$ is a 
standard automorphism of $F(H)$. Hence, $A \theta \subseteq B \theta$. By Theorem 4, $\theta$ is a standard automorphism of $F(G)$.

CoRollary 2. If $P$ denotes the set of prime integers and $q \in P$, then Aut $F\left(Z\left(q^{\infty}\right)\right)$ is isomorphic to Aut $Z\left(q^{\infty}\right)$ and Aut $F(Q / Z)$ is isomorphic to $\Pi_{p \in P}$ Aut $F\left(Z\left(p^{\infty}\right)\right)$.

Proof. By [2, p. 221-222], $Q / Z$ is isomorphic to $\sum_{p \in P} Z\left(p^{\infty}\right)$ and Aut $Q / Z$ is isomorphic to $\Pi_{p \in P}$ Aut $Z\left(p^{\infty}\right)$. With these observations the corollary is an immediate consequence of the theorem.

\section{REFERENCES}

1. R.D. Byrd, J. T. Lloyd, F. D. Pedersen. and J. W. Stepp, Automorphisms of the semigroup of finite complexes of a torsion-free abelian group, submitted.

2. L. Fuchs, Abelian Groups, Pergamon Press, New York, 1960.

3. H. B. Mann, On products of sets of group elements, Canad. J. Math., 4 (1952), 64-66.

Received March 25, 1977

UNIVERSITY OF HOUSTON

Houston, TX 77004

AND

SOUTHERN ILLINOIS UNIVERSITY

Carbondale, IL 62901 



\title{
PACIFIC JOURNAL OF MATHEMATICS
}

\author{
EDITORS
}

RICHARD ARENS (Managing Editor)

University of California

Los Angeles, California 90024

C. W. CurTis

University of Oregon

Eugene, OR 97403

C. C. MOORE

University of California

Berkeley, CA 94720
J. DUgundJI

Department of Mathematics

University of Southern Californı

Los Angeles, California 90007

R. Finn and J. Milgram

Stanford University

Stanford, California 94305

\section{ASSOCIATE EDITORS}

E. F. BECKENBACH

\section{SUPPORTING INSTITUTIONS}

\author{
UNIVERSITY OF BRITISH COLUMBIA \\ CALIFORNIA INSTITUTE OF TECHNOLOGY \\ UNIVERSITY OF CALIFORNIA \\ MONTANA STATE UNIVERSITY \\ UNIVERSITY OF NEVADA, RENO \\ NEW MEXICO STATE UNIVERSITY \\ OREGON STATE UNIVERSITY \\ UNIVERSITY OF OREGON \\ OSAKA UNIVERSITY
}

UNIVERSITY OF SOUTHERN CALIFORNIA

STANFORD UNIVERSITY

UNIVERSITY OF TOKYO

UNIVERSITY OF UTAH

WASHINGTON STATE UNIVERSITY

UNIVERSITY OF WASHINGTON
AMERICAN MATHEMATICAL SOCIETY
NAVAL WEAPONS CENTER 


\section{Pacific Journal of Mathematics \\ Vol. 72 , No. 1 \\ January, 1977}

Kazuo Anzai and Shiro Ishikawa, On common fixed points for several

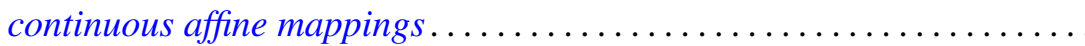

Bruce Alan Barnes, When is a representation of a Banach $*$-algebra

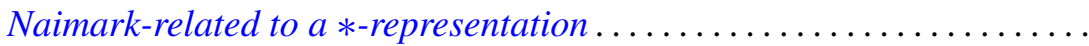

Richard Dowell Byrd, Justin Thomas Lloyd, Franklin D. Pedersen and

James Wilson Stepp, Automorphisms of the semigroup of finite

complexes of a periodic locally cyclic group ...................

Donald S. Coram and Paul Frazier Duvall, Jr., Approximate fibrations and a

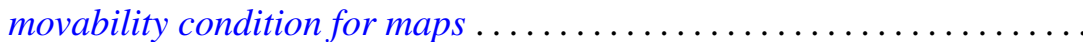

Kenneth R. Davidson and Che-Kao Fong, An operator algebra which is not

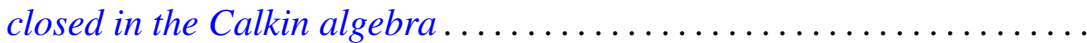

Garret J. Etgen and James Pawlowski, A comparison theorem and oscillation criteria for second order differential systems .............

Philip Palmer Green, $C^{*}$-algebras of transformation groups with smooth

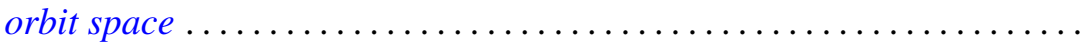

Charles Allen Jones and Charles Dwight Lahr, Weak and norm approximate

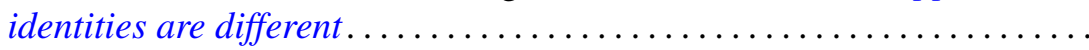

G. K. Kalisch, On integral representations of piecewise holomorphic

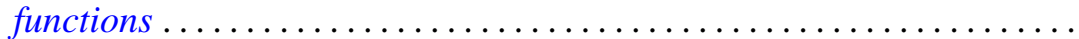

Y. Kodama, On product of shape and a question of Sher

Heinz K. Langer and B. Textorius, On generalized resolvents and

$Q$-functions of symmetric linear relations (subspaces) in Hilbert

space ...................................

Albert Edward Livingston, On the integral means of univalent, meromorphic functions

Wallace Smith Martindale, III and Susan Montgomery, Fixed elements of

Jordan automorphisms of associative rings ..........

R. Kent Nagle, Monotonicity and alternative methods for nonlinear boundary value problems ........................

Richard John O'Malley, Approximately differentiable functions: the $r$ topology.

Mangesh Bhalchandra Rege and Kalathoor Varadarajan, Chain conditions

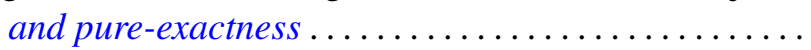

Christine Ann Shannon, The second dual of $C(X)$. .

Sin-ei Takahasi, A characterization for compact central double centralizers of $C^{*}$-algebras .

Theresa Phillips Vaughan, A note on the Jacobi-Perron algorithm. . .

Arthur Anthony Yanushka, A characterization of $\operatorname{PSp}(2 m, q)$ and

$\mathrm{P} \Omega(2 m+1, q)$ as rank 3 permutation groups ......... 